\title{
Control of Structural Coloration by Natural Sunlight Irradiation on a Melanin Precursor Polymer Inspired by Skin Tanning
}

Taku Okoshi†, Takeshi Iwasaki ${ }^{\dagger,}$, Shimon Takahashi ${ }^{\dagger}$, Yasuhiko Iwasaki§, ${ }^{\S}$, Keiki Kishikawa ${ }^{\dagger}$, and Michinari Kohri*,†

†Department of Applied Chemistry and Biotechnology, Graduate School of Engineering, Chiba University, 1-33 Yayoi-cho, Inage-ku, Chiba 263-8522, Japan

*Banknote Department, Head Office, National Printing Bureau, 2-2-5 Toranomon, Minato-ku, Tokyo 105-8445, Japan

§ORDIST, Kansai University, 3-3-35 Yamate-cho, Suita-shi, Osaka, 564-0836, Japan

${ }^{\perp}$ Faculty of Chemistry, Materials and Bioengineering, Kansai University, 3-3-35 Yamate-cho, Suita-shi, Osaka, 564-0836, Japan

\section{Corresponding Author}

*E-mail address: kohri@faculty.chiba-u.jp (M. K.) 
(a)

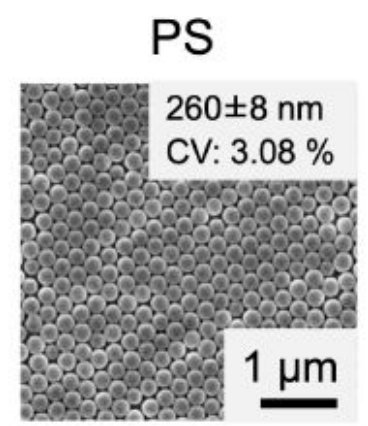

(b)

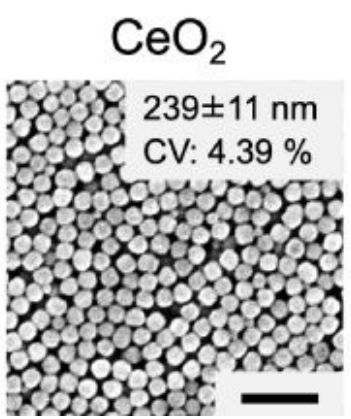

PS UV(3)

$227 \pm 9 \mathrm{~nm}$

CV: $4.12 \%$

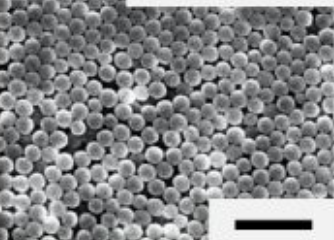

$\mathrm{CeO}_{2} \cup \mathrm{U}(3)$

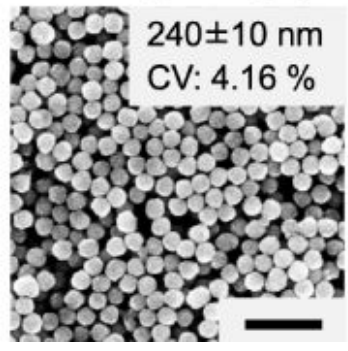

Figure S1. SEM images of (a) PS particles and (b) $\mathrm{CeO}_{2}$ particles before and after UV light irradiation $(254 \mathrm{~nm}, 3 \mathrm{~h})$. The numbers in the figure are the average diameter and coefficient of variation $(\mathrm{CV})$ of the particles calculated from 100 particles obtained from the SEM image.

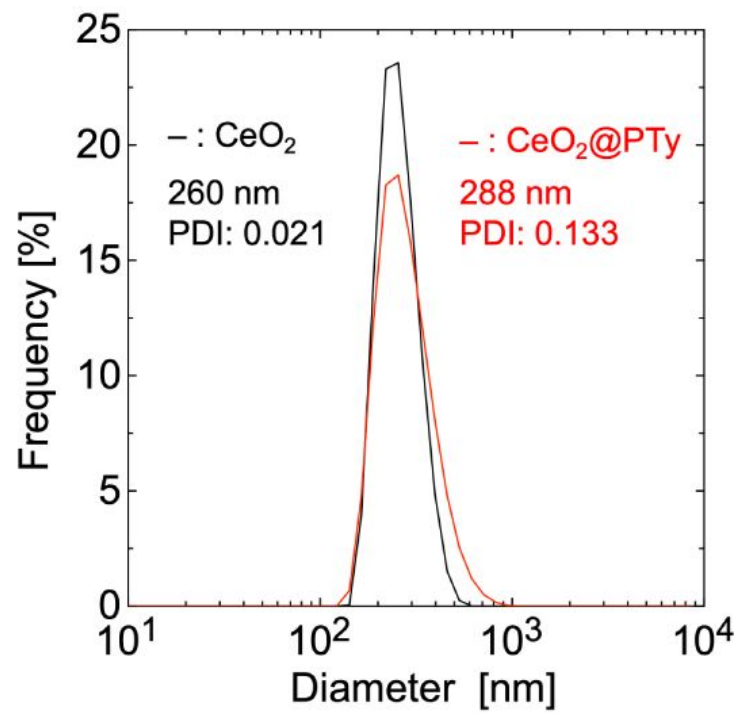

Figure S2. Size distributions measured by DLS of a water dispersed solution of $\mathrm{CeO}_{2}$ (black line) and (b) $\mathrm{CeO}_{2} @$ PTy particles (red line). The numbers in the figure are hydrodynamic diameter and polydispersity index (PDI). 

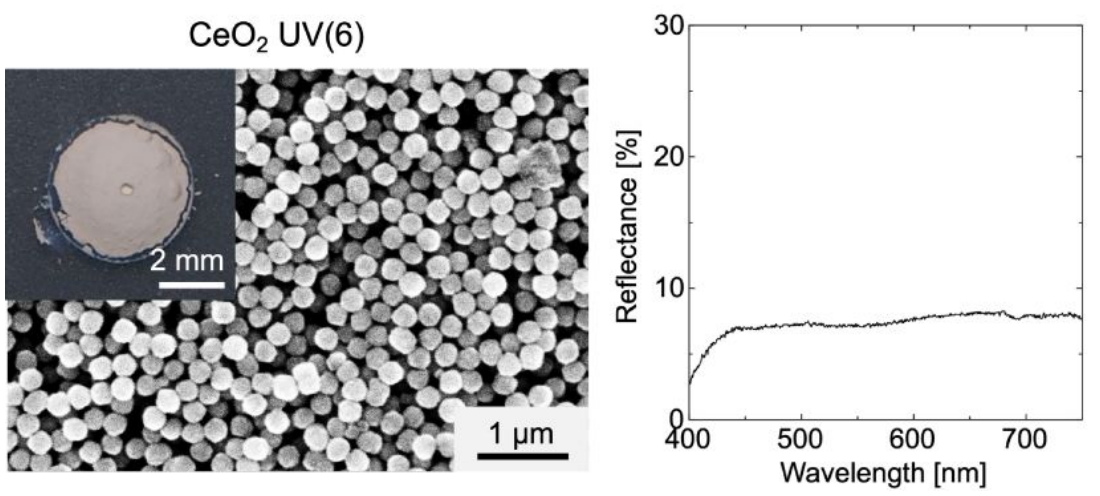

Figure S3. Photograph, SEM image, and reflection spectrum of pellet sample from $\mathrm{CeO}_{2} \mathrm{UV}(6)$ particles.
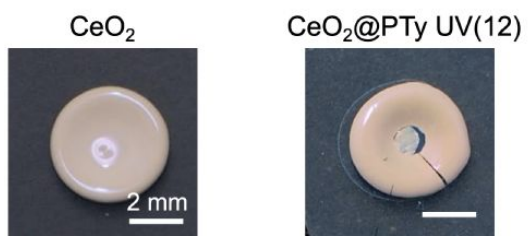

Figure S4. Photographs of pellet samples from $\mathrm{CeO}_{2}$ and $\mathrm{CeO}_{2} @ \mathrm{PTy} \mathrm{UV}(12)$ particles.

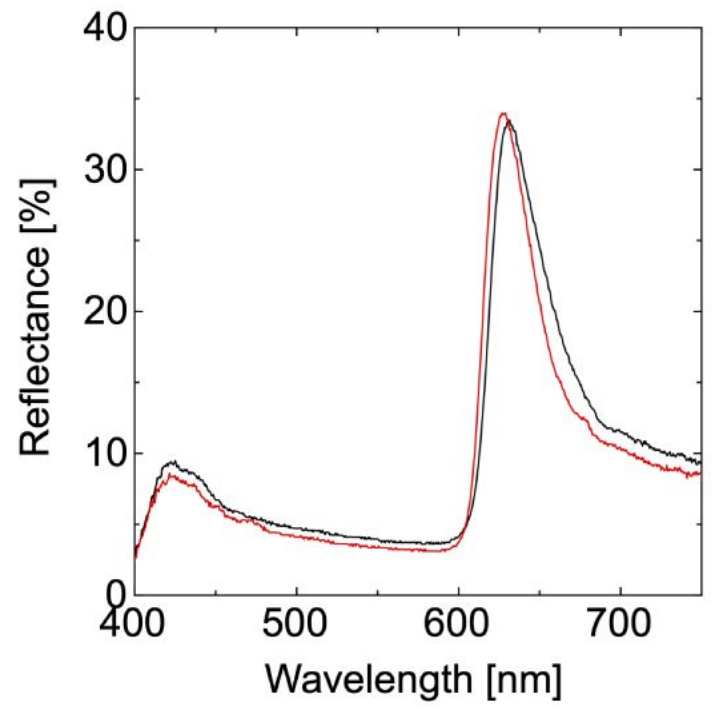

Figure S5. Reflection spectra of pellet samples of $\mathrm{CeO}_{2} @$ PTy UV(6) particles. The black line is the spectrum of the sample immediately after preparation, and the red line is the spectrum of the same pellet sample stored for 7 months. 
(a)
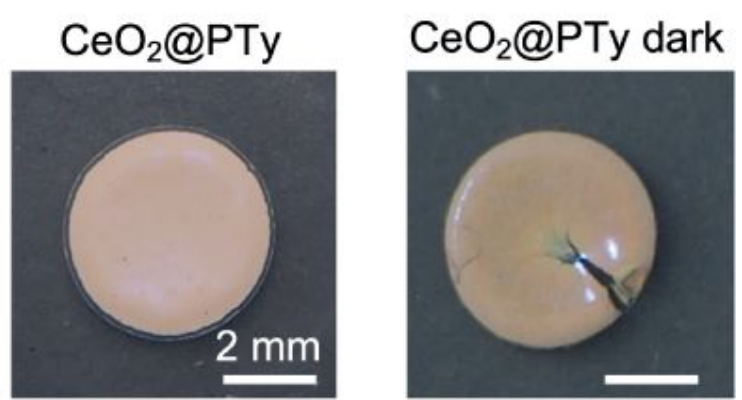

\section{$\mathrm{CeO}_{2} @ P T y$ dark UV}

(b)

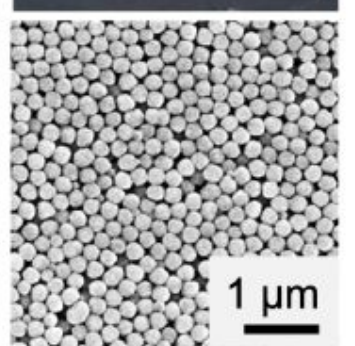

(c)

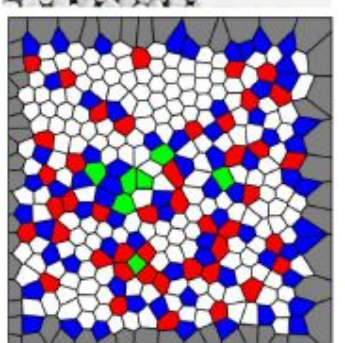

(d)
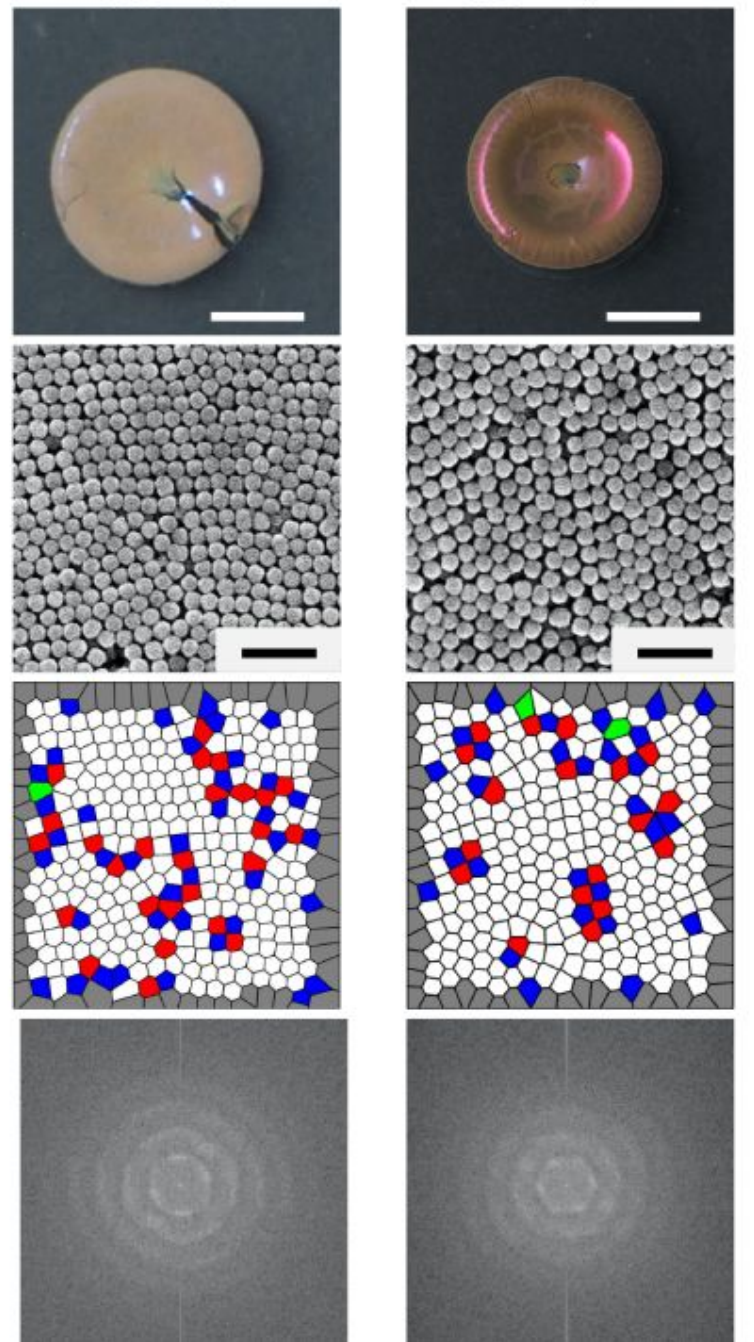

Figure S6. (a) Photographs and (b) SEM images of the pellet samples prepared from $\mathrm{CeO}_{2} @ \mathrm{PTy}$, $\mathrm{CeO}_{2} @$ PTy dark, and $\mathrm{CeO}_{2} @$ PTy dark UV particles. (c) The Voronoi diagrams of SEM images shown in (b). The Voronoi cells on the boundary of analyzed square region (dark gray) are not included in this analysis. Blue: five-side polygon, white: hexagonal polygon, red: seven-sided polygon, and green: other. (d) The Fourier transform spectra from SEM images shown in (b). 


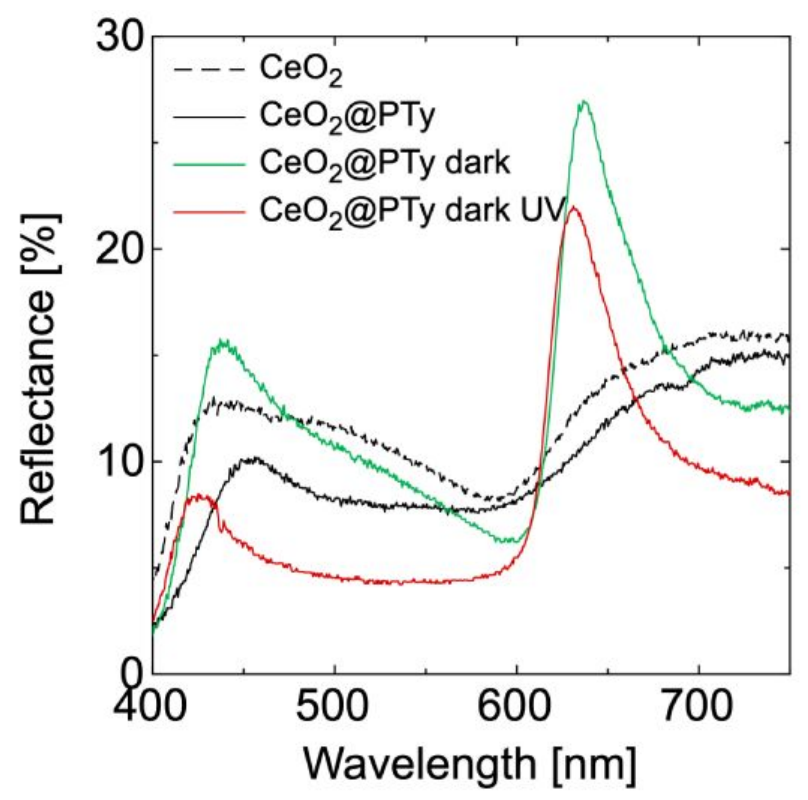

Figure S7. Reflection spectra of pellet samples from $\mathrm{CeO}_{2}, \mathrm{CeO}_{2} @ \mathrm{PTy}, \mathrm{CeO}_{2} @$ PTy dark, and $\mathrm{CeO}_{2} @$ PTy dark UV particles.
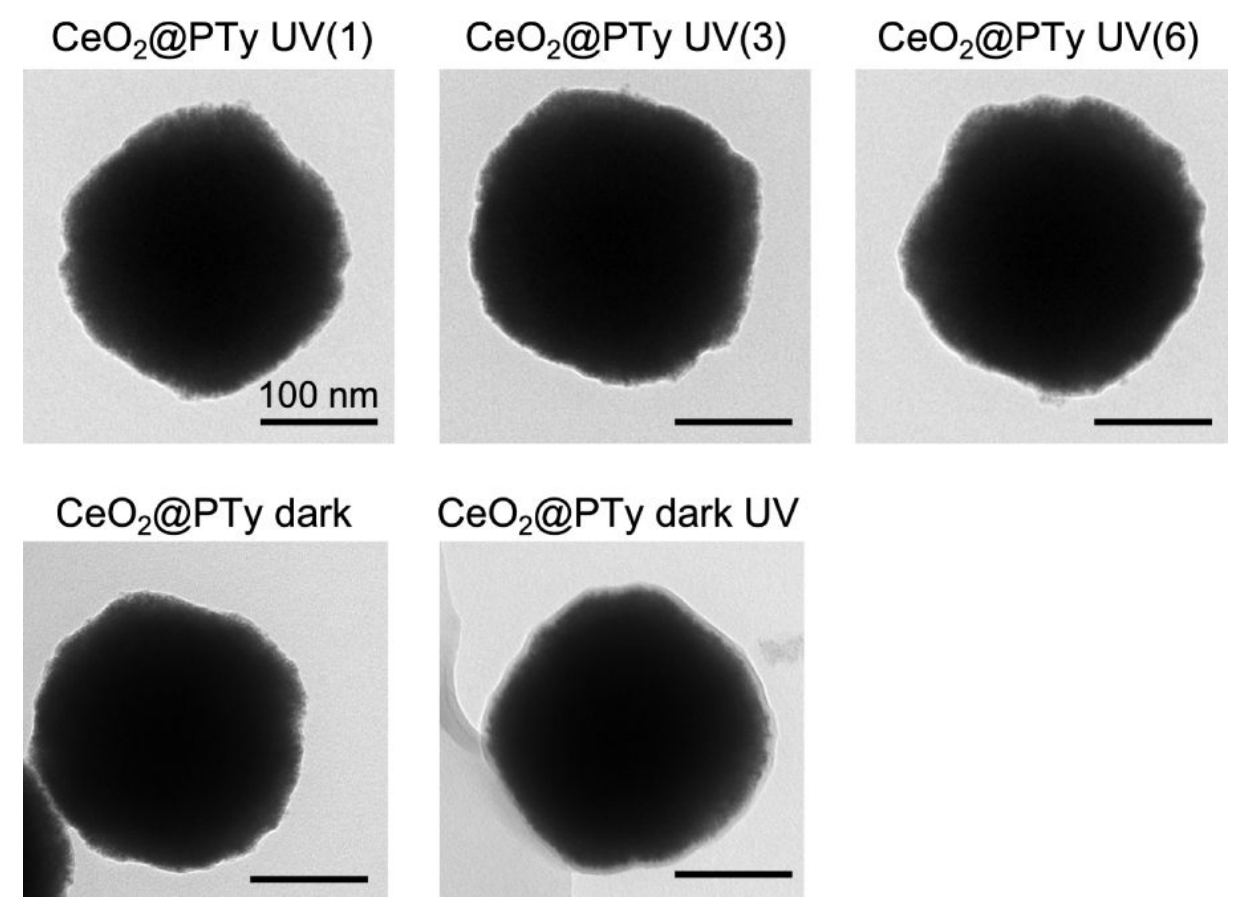

Figure S8. TEM images of the $\mathrm{CeO}_{2} @$ PTy UV $(t), \mathrm{CeO}_{2} @$ PTy dark, and $\mathrm{CeO}_{2} @$ PTy dark UV particles 

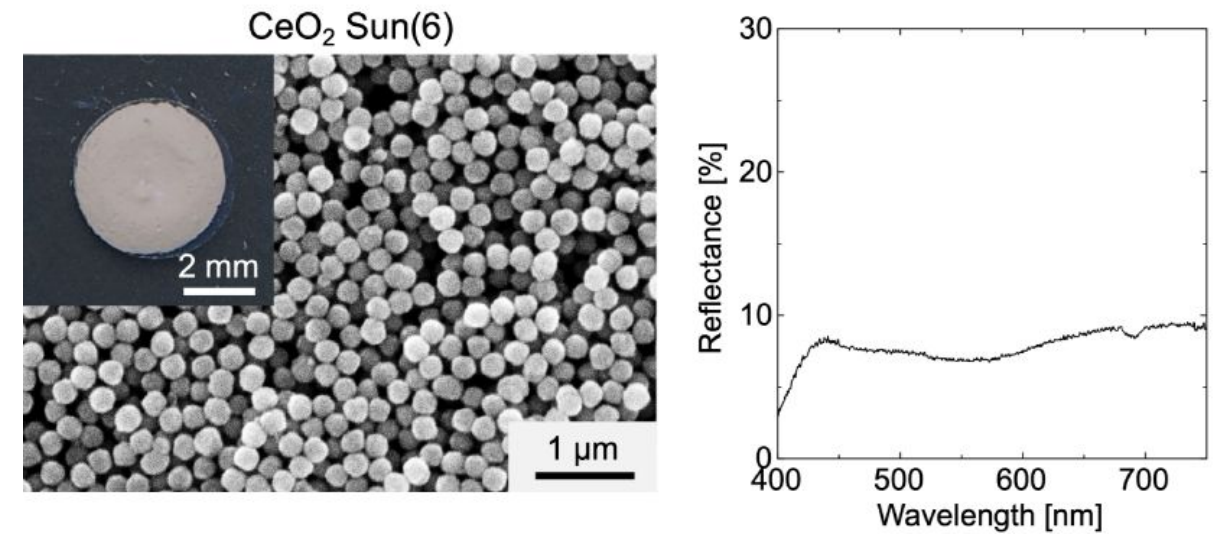

Figure S9. Photograph, SEM image and reflection spectrum of pellet sample from $\mathrm{CeO}_{2} \mathrm{Sun}(6)$ particles.

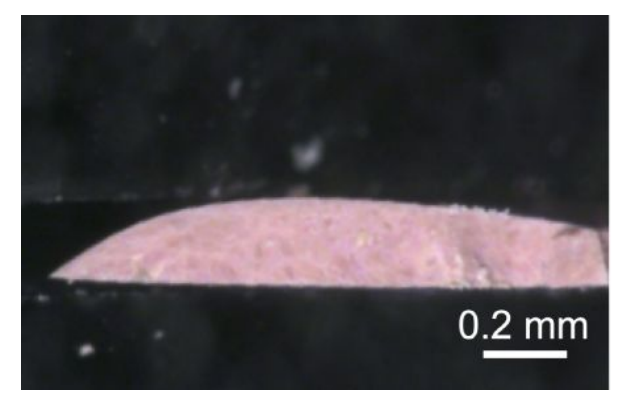

Figure S10. Cross-sectional photograph of the pellet sample of $\mathrm{CeO}_{2} @$ PTy UV(3) particles. 


\section{(a) $\mathrm{CeO}_{2} @$ PTy Sun(1)}
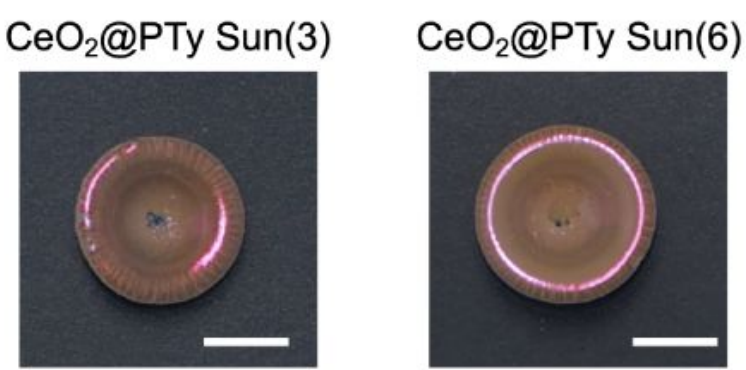

(b)
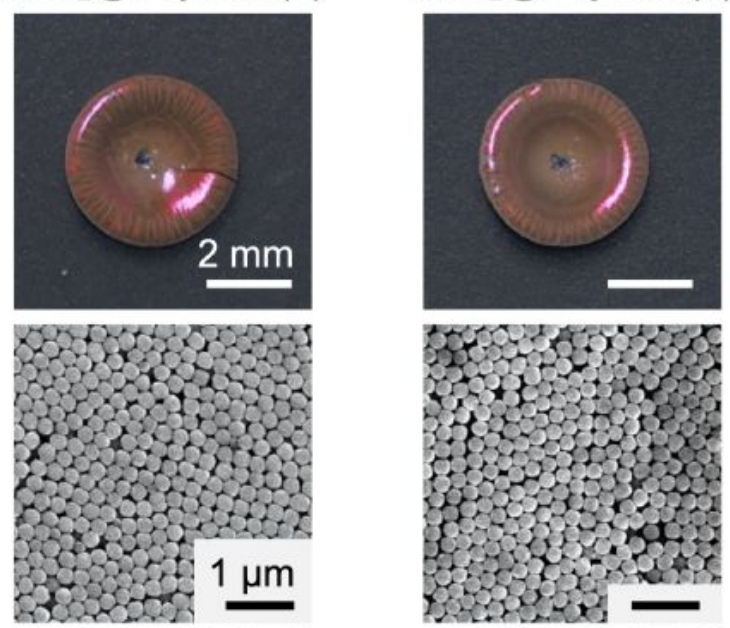

(c)
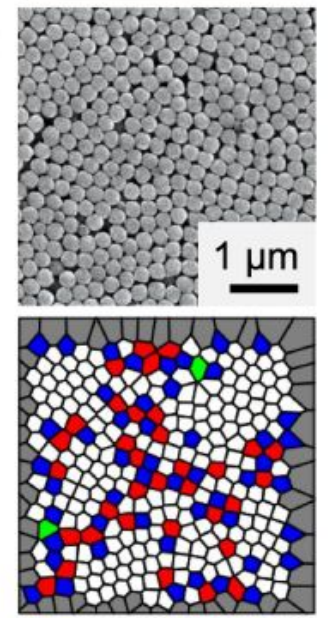

(d)
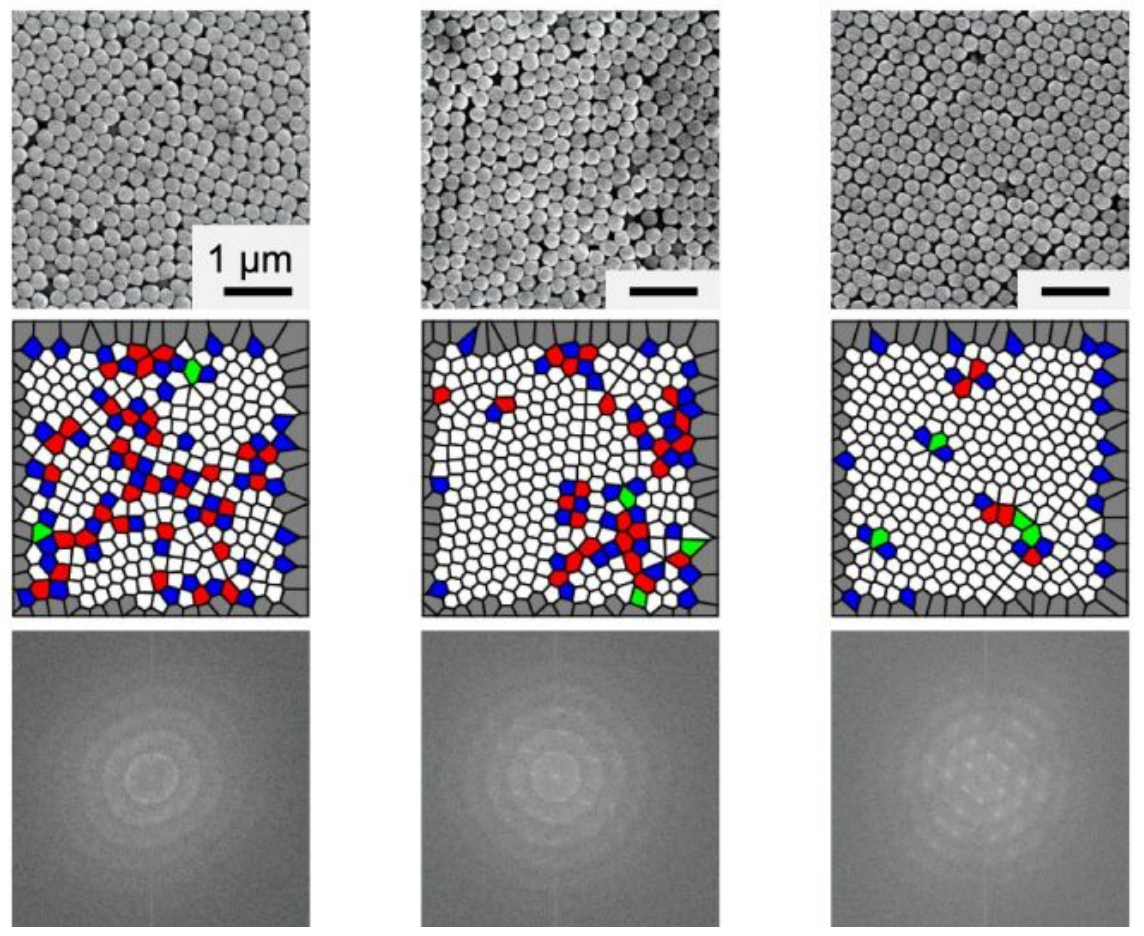

Figure S11. (a) Photographs and (b) SEM images of the pellet samples prepared from $\mathrm{CeO}_{2} @ \mathrm{PTy}$ $\operatorname{Sun}(t)$ core-shell particle. (c) The Voronoi diagrams of SEM images shown in (b). The Voronoi cells on the boundary of analyzed square region (dark gray) are not included in this analysis. Blue: five-side polygon, white: hexagonal polygon, red: seven-sided polygon, and green: other. (d) The Fourier transform spectra from SEM images shown in (b). 


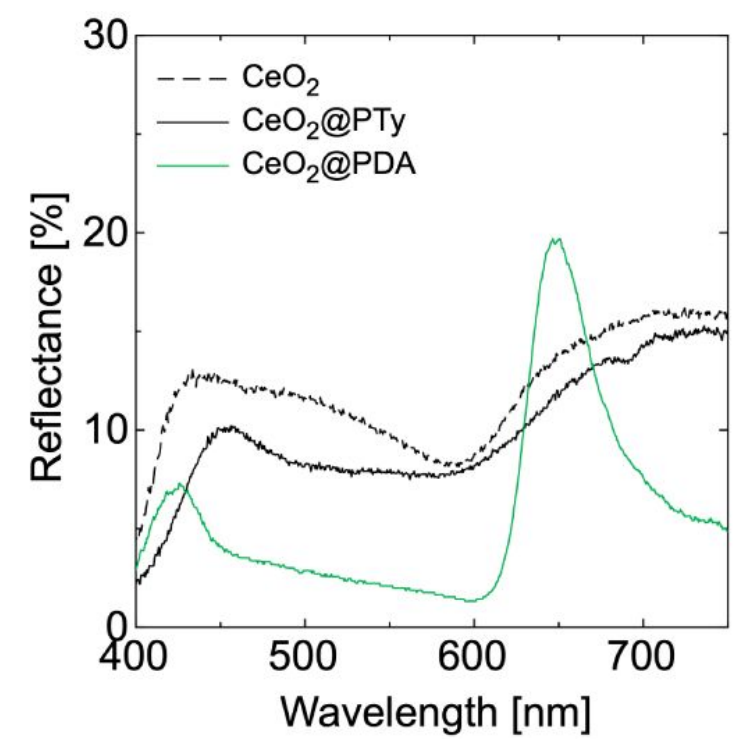

Figure S12. Reflection spectra of pellet samples from $\mathrm{CeO}_{2}, \mathrm{CeO}_{2} @ \mathrm{PTy}$, and $\mathrm{CeO}_{2} @ \mathrm{PDA}$ particles. 